\title{
Angular Momentum Flow during Ultrafast Demagnetization of a Ferrimagnet
}

\author{
Martin Hennecke, ${ }^{1}$ Ilie Radu, ${ }^{1, *}$ Radu Abrudan, ${ }^{2}$ Torsten Kachel, ${ }^{2}$ Karsten \\ Holldack, ${ }^{2}$ Rolf Mitzner, ${ }^{2}$ Arata Tsukamoto ${ }^{3}$ and Stefan Eisebitt ${ }^{1,4}$ \\ ${ }^{1}$ Max-Born-Institut für Nichtlineare Optik und Kurzzeitspektroskopie, \\ Max-Born-Straße 2A, 12489 Berlin, Germany \\ ${ }^{2}$ Helmholtz-Zentrum Berlin für Materialien und Energie, \\ Albert-Einstein-Straße 15, 12489 Berlin, Germany \\ ${ }^{3}$ College of Science and Technology, \\ Nihon University, 7-24-1 Funabashi, Chiba, Japan \\ ${ }^{4}$ Institut für Optik und Atomare Physik, Technische Universität Berlin, \\ Straße des 1\%. Juni 135, 10623 Berlin, Germany
}

(Dated: March 18, 2019)

\begin{abstract}
One of the key processes setting the speed of ultrafast magnetization phenomena is the angular momentum transfer from and into the spin system. However, the way the angular momentum flows during ultrafast demagnetization and magnetization switching phenomena remains elusive so far. We report on time-resolved soft X-ray Magnetic Circular Dichroism (XMCD) measurements of ferrimagnetic GdFeCo alloy allowing us to record the dynamics of elemental spin and orbital moments at the Fe and Gd sites during femtosecond laser-induced demagnetization. We observe a complete transfer of spin and orbital angular momentum to the lattice during the first hundreds of femtoseconds of the demagnetization process.
\end{abstract}


Since Beaurepaire et al. discovered in 1996 that ferromagnetic nickel can be demagnetized on a sub-picosecond time scale by femtosecond (fs) laser pulse excitations [1], the investigation of ultrafast magnetization dynamics has become an intense field of research. Because the observed demagnetization time scale was several orders of magnitude faster than for manipulating magnetization via application of external magnetic fields [2], this topic became not only relevant for fundamental science, but also for applications such as future magnetic data storage technologies [3]. Recent studies of ferrimagnetic GdFeCo alloys have revealed an ultrafast laser-induced magnetization reversal mediated by a transient ferromagnetic-like state [4]; such switching was purely thermally-driven without the need of any other external stimulus, relying on the exchange coupling between the RE and TM sublattices [5]. However, one of the key aspects involved in these processes still remains unclear, namely how the ultrafast angular momentum transfer from and into the spin system is happening during demagnetization and switching events $[6,7]$.

Although it is widely accepted that, ultimately, the lattice should act as the final recipient of angular momentum, like evidenced macroscopically in the Einstein-De Haas effect [8], the spin-lattice angular momentum exchange mechanism at the microscopic level is actually unknown. For instance, it is still an open question whether the magnetization is reduced due to a direct transfer of angular momentum from the spin system to the lattice or if the orbital moment of the electrons has a dominant role instead, be it as a transient reservoir of angular momentum and/or as the pivotal means to couple to the lattice. Recent element-specific demagnetization investigations of elemental $\mathrm{Ni}[7]$ and Co-based alloys [9, 10] interpreted their findings within the framework of these two scenarios: a direct spin-lattice mechanism has been invoked in the case of $\mathrm{Ni}$ while for $\mathrm{CoPd}$ and $\mathrm{Co}(\mathrm{Gd}, \mathrm{Tb})$ alloys a faster orbital moment dynamics compared to its spin counterpart has been observed, presumably due to a laser-induced quenching of the magneto-crystalline anisotropy. Although these aforementioned fs X-ray Magnetic Circular Dichroism (XMCD) studies were the first to provide separate information on the dynamics of spin and orbital moments in magnetic materials, they provide no definitive answers regarding the microscopic mechanism responsible for the ultrafast dissipation and exchange of angular momentum.

In this work, we investigate the path of angular momentum flow during laser-driven ultrafast demagnetization of ferrimagnetic GdFeCo alloy. Via the time- and element-resolved XMCD measurements we are able to disentangle the transient dynamics of spin and orbital 
magnetic moments individually at the Fe and Gd sites. Our data provide strong evidence for a lack of inter-atomic angular momentum exchange between Fe and Gd; furthermore, within the experimental time resolution of $130 \mathrm{fs}$, we do not observe any transient increase or accumulation of angular momentum in the orbital momentum degree of freedom of Fe, as mediated by a potential intra-atomic angular momentum transfer between the spin $(S)$ and orbital $(L)$ momenta. As shown below, these findings are in line with a recently reported mechanism where spin angular momentum is transferred intra-atomically to orbital angular momentum via spin-orbit coupling on a time scale of tens of fs (proportional to the spin-orbit coupling strength), while orbital moment, in turn, is continuously dumped into the lattice on a much faster time scale of the order of $1 \mathrm{fs}$ [11].

Time-resolved XMCD measurements were performed at the FemtoSpeX fs-slicing facility of the BESSY II synchrotron light source, which provides 100 fs full width at half maximum (FWHM) soft X-ray pulses with circular polarization [12]. Soft X-ray radiation is required for measuring the XMCD at $\mathrm{Fe}_{2,3}$ and $\mathrm{Gd} \mathrm{M}_{4,5}$ absorption edges, probing the dynamics of the $3 \mathrm{~d}$ magnetic moment of Fe and the $4 \mathrm{f}$ magnetic moment of Gd, respectively. The demagnetization process was driven by linearly polarized optical pulses of $40 \mathrm{fs}$ FWHM, generated by an $800 \mathrm{~nm}$ pump laser system. The time-resolved data were acquired in a transmission geometry, employing a pump-probe-scheme. Exciting the sample with a repetition rate of $3 \mathrm{kHz}$ while probing with $6 \mathrm{kHz}$ allowed us to simultaneously measure the optically pumped and unpumped state of the system as a function of pump-probe delay, using a gated photodetection system. The incident pump laser fluence used for the pump-probe measurements was $12.9 \mathrm{~mJ} / \mathrm{cm}^{2}$ unless otherwise stated (for more experimental details, see Supplementary Information).

The sample studied was a $20 \mathrm{~nm}$ thick amorphous film of a ferrimagnetic $\mathrm{Gd}_{25.3} \mathrm{Fe}_{65.4} \mathrm{Co}_{9.3}$ alloy deposited on a $500 \mathrm{~nm}$ thick $\mathrm{Al}$ foil and sandwiched between two $\mathrm{Si}_{3} \mathrm{~N}_{4}$ layers. The exact sample composition is $\mathrm{Si}_{3} \mathrm{~N}_{4}(60) / \mathrm{GdFeCo}(20) / \mathrm{Si}_{3} \mathrm{~N}_{4}(5) / \mathrm{AlTi}(10) / \mathrm{Al}(500)$. A small amount of Co was added to the magnetic film in order to increase the out-of-plane magnetic anisotropy. Fe and Co are ferromagnetically coupled and thus forming a transition metal sublattice of FeCo which is ferrimagnetically coupled to the rare-earth sublattice of Gd with a magnetization compensation temperature of $250 \mathrm{~K}$. Atom-specific XMCD measurements were carried out for Gd and Fe. All measurements were done at room temperature well above the magnetization compensation point. In Fig. 1, we show the static X-ray absorption 

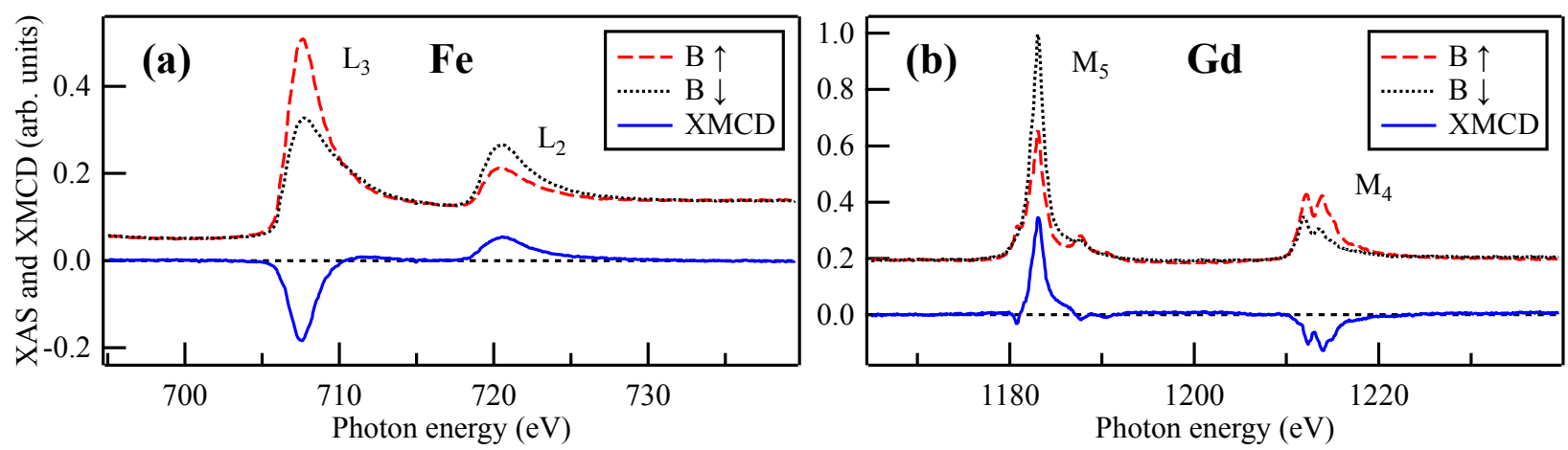

FIG. 1 (Color online). Static soft X-ray absorption (XAS) and XMCD spectra of the studied GdFeCo sample measured in the energy range of (a) $\mathrm{Fe} \mathrm{L}_{2,3}$ and (b) $\mathrm{Gd} \mathrm{M}_{4,5}$ absorption edges. The XAS spectra (red and black) were recorded with circularly polarized X-ray light by using an out-of-plane magnetic field $B$ to saturate the sample in opposite directions. Taking the difference of both spectra leads to the XMCD spectrum (blue). The measurements were carried out with an energy resolution of $0.1 \mathrm{eV}$ using ALICE reflectometer [13] at the PM3 beamline of BESSY II. [14]

spectra (XAS) of the sample measured for opposite magnetic field directions at the $\mathrm{L}_{2,3}$ edges of Fe and $\mathrm{M}_{4,5}$ edges of $\mathrm{Gd}$ and the difference spectra, corresponding to the XMCD. For the time-resolved measurements, the photon energy was tuned to the maximum XMCD magnitude for the element under investigation.

Measuring time-resolved XMCD data at both $\mathrm{L}_{2,3}$ and $\mathrm{M}_{4,5}$ absorption edges of $\mathrm{Fe}$ and Gd allows us to disentangle the spin and orbital contributions for each element via magnetooptical sum rules. The sum rules relate $S$ and $L$ moments to the spectral intensities integrated over the respective XAS and XMCD spectra and can be derived as shown in [15-18] for both $3 \mathrm{~d}$ transition metals and rare-earth elements. Note that the sum rules were shown to be valid even for the highly non-equilibrium states generated by intense laser excitation of the electronic system [19] (see Supplementary Information).

In Fig. 2, the measured time evolution of the XMCD at $\mathrm{Fe}_{3,2}$ and $\mathrm{Gd}_{5,4}$ absorption edges is shown as a function of pump-probe delay. As expected [4], the Fe and Gd sublattices show different demagnetization dynamics. In order to retrieve the time constants and magnitude of the demagnetization process, the XMCD data was fitted using a single 

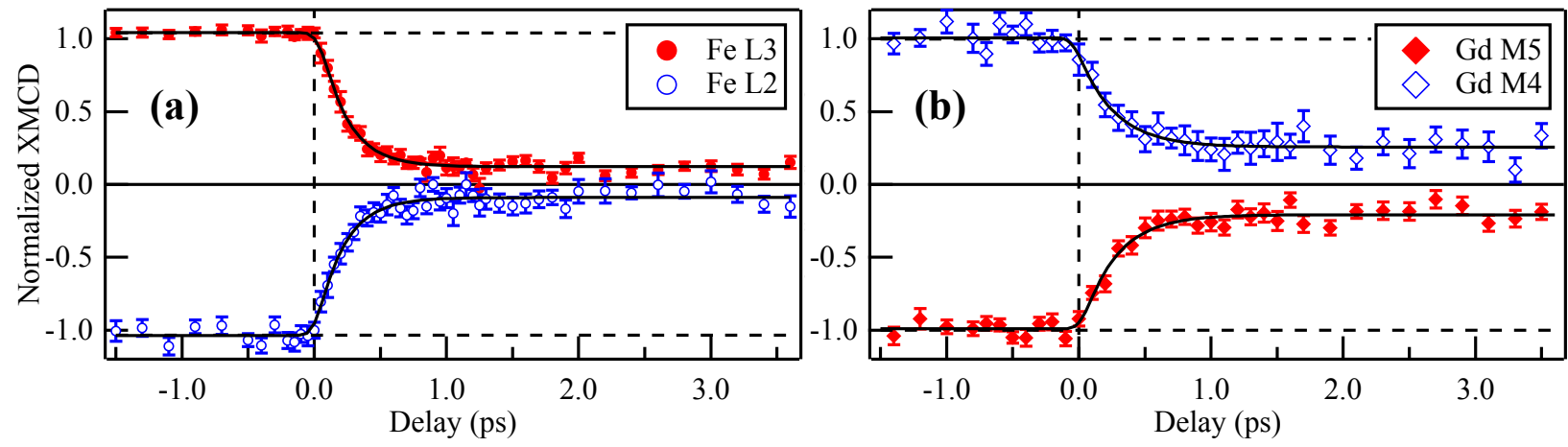

FIG. 2 (Color online). Time-resolved XMCD at the (a) Fe $\mathrm{L}_{2,3}$ and (b) Gd $\mathrm{M}_{4,5}$ absorption edges of GdFeCo measured as a function of pump-probe delay. The temporal resolution of the measurement is $\approx 130 \mathrm{fs}$. The data is normalized to the unpumped XMCD signal and fitted using a single exponential function (black solid lines). The error bars are calculated as the standard error of the mean.

exponential fit function to describe the ultrafast demagnetization process:

$$
f(t)=g(t) \otimes \begin{cases}A, & t \leq 0 \\ A-B\left[1-\exp \left(-\frac{t}{\tau_{\mathrm{D}}}\right)\right], & t>0\end{cases}
$$

where $\tau_{\mathrm{D}}$ is the time constant for demagnetization, while $A$ describes the unpumped XMCD signal at negative delays with $B$ corresponding to the amplitude of the exponential decay. By convolution with a Gaussian function $g(t)$, the time resolution of $\approx 130 \mathrm{fs}(\mathrm{FWHM})$ is taken into account. We observe that the Fe sublattice demagnetizes with a time constant of $\tau_{\mathrm{L}_{3}}(\mathrm{Fe})=201 \pm 15$ fs and a pump-induced maximum change in XMCD of $88 \pm 1 \%$ measured at the $\mathrm{L}_{3}$ absorption edge, and $\tau_{\mathrm{L}_{2}}(\mathrm{Fe})=204 \pm 24 \mathrm{fs}$ and $91 \pm 2 \%$ at the $\mathrm{L}_{2}$ edge respectively. The demagnetization of the Gd sublattice takes longer with a time constant of $\tau_{\mathrm{M}_{5}}(\mathrm{Gd})=259 \pm 42 \mathrm{fs}$ and a pump-induced change in XMCD of $79 \pm 2 \%$ measured at the $\mathrm{M}_{5}$ absorption edge, and $\tau_{\mathrm{M}_{4}}(\mathrm{Gd})=270 \pm 54 \mathrm{fs}$ and $75 \pm 2 \%$ at the $\mathrm{M}_{4}$ edge. For each element, both independently determined time constants agree within the experimental error and show a significantly different demagnetization speed at Fe vs. Gd sites with the transition metal sublattice exhibiting faster demagnetization. This observation is in agreement with the values reported in the literature for GdFe and GdCo alloys measured above their compensation temperatures $[10,20]$. The different demagnetization time of the elements have been shown to be related both to the magnitude of the elemental magnetic moments in the alloy (see 

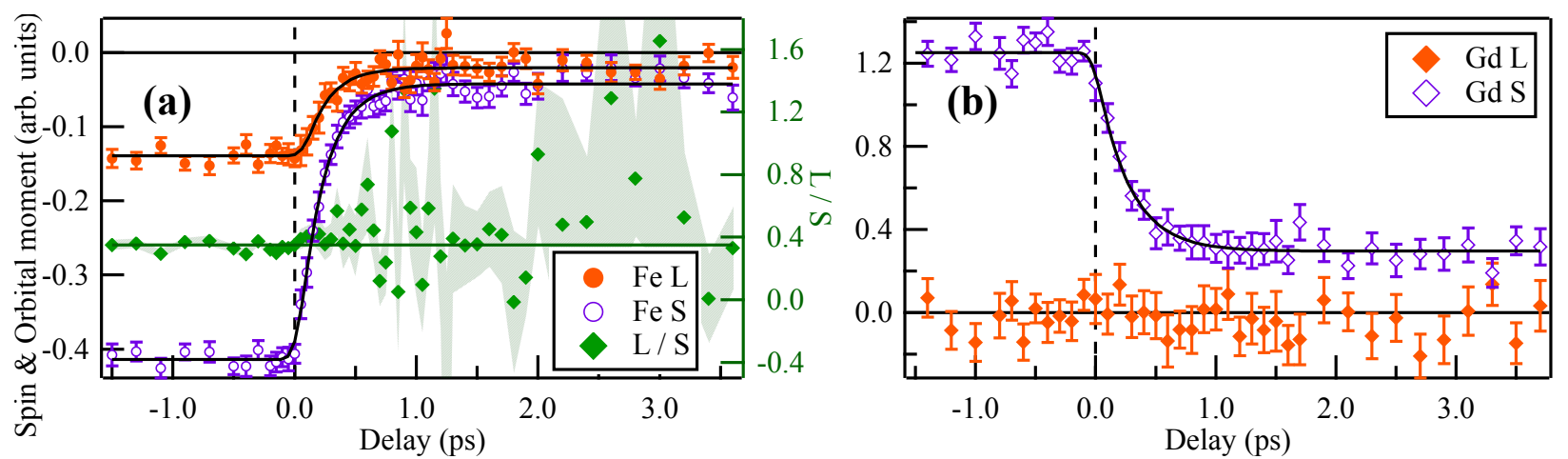

FIG. 3 (Color online). Time-resolved evolution of spin and orbital moment at Fe and Gd sites during demagnetization extracted by application of sum rules from the XMCD data shown in Fig. 2. (a) At Fe sites the demagnetization occurs due to a decrease of both spin and orbital moments, equal in magnitude and speed, leading to a constant $L / S$ ratio (green) during the demagnetization process. (b) At Gd sites, the demagnetization is purely driven by a decrease of spin moment, while the orbital moment stays zero at all times; implicitly, the $\mathrm{Gd} L / S$ ratio remains also zero and is not shown in the figure. The error bars and shaded areas correspond to the error propagation of the standard error of the mean.

e.g. Ref. $[4,20])$ as well as to the equilibrium Curie temperature of the sample [21]. According to Ref. [21], laser-generated (lattice) temperatures in the close proximity of the Curie point $\left(T / T_{C}=0.8\right)$ can lead to faster demagnetization dynamics of the rare-earth sublattice, thus approaching the demagnetization speed of the transition metal sublattice. Results of two-temperature model (2TM) simulations of GdFe, mimicking the experimental conditions of our time-resolved XMCD measurements, show that this temperature regime is indeed reached within 300-500 fs after laser excitation, with a electron-phonon thermalization time of $\approx 1 \mathrm{ps}$ (for the output of the $2 \mathrm{TM}$ simulations, see Supplementary Information). This theoretical prediction is corroborated by our observation of slightly faster demagnetization times measured for Gd compared to previous experimental results reported in Ref. [10, 20].

Via the sum rules, we disentangle the dynamics of spin and orbital moments at the Fe and Gd atoms. Their dynamics are shown in Fig. 3. At Gd sites, we observe a demagnetization that is purely driven by a decrease of spin moment with a time constant of $\tau_{S}(\mathrm{Gd})=$ $269 \pm 32 \mathrm{fs}$, while the orbital moment is zero at all times and not participating during the demagnetization process. While this is expected for $\mathrm{Gd}$ under quasi-equilibrium conditions 
due to its half-filled 4 f shell that leads to $L=0$, the data show that there is also no transient orbital moment induced by laser excitation or via transfer of angular momentum during demagnetization. Thus, within our experimental time resolution, the ratio $L / S$ remains zero at all times and any transfer of angular momentum from or to Gd orbital moment during the measured time scales (up to 4 ps) can be ruled out; a similar behavior has been reported for the Gd sublattice in ferrimagnetic GdCo alloys in Ref. [10], where a sum rules analysis was carried out for Gd and Co sublattices. At Fe sites, the demagnetization is due to a decrease of both spin and orbital moment. Fitting the data yields the same time constants within the experimental error for both moments, namely $\tau_{S}(\mathrm{Fe})=202 \pm 13 \mathrm{fs}$ and $\tau_{L}(\mathrm{Fe})=193 \pm 42 \mathrm{fs}$. The ratio $L / S$ at the Fe atoms stays also constant during demagnetization. This finding implies that, within our experimental accuracy, both spin and orbital moments decrease equally in magnitude and demagnetize with the same speed. This behavior is different from XMCD results on the transition metal sublattices of Co in CoGd and CoPd reported earlier $[9,10]$. These latter reports showed a slightly faster and more pronounced orbital momentum quench compared to the spin moment, leading to a transient change of the ratio $L / S$. This behavior is not present in our case for the Fe sublattice and can be ascribed to the stronger spin-orbit coupling and magneto-crystalline anisotropy present in Co with respect to $\mathrm{Fe}$ [22]. It is worth mentioning here that bcc Fe has the smallest spin-orbit coupling constant among the $3 \mathrm{~d}$ ferromagnets $\mathrm{Ni}$, Co and Fe: the calculated s-o coupling constants are $70 \mathrm{meV}$, $90 \mathrm{meV}$ and $110 \mathrm{meV}$ for $\mathrm{Fe}, \mathrm{Co}$ and $\mathrm{Ni}$, respectively [11, 22]. From the transient dynamics of $L$ and $S$ at Fe sites we can conclude that, within our time resolution of $130 \mathrm{fs}$, there is no transient increase or accumulation of angular momentum in the orbital moment of Fe. Note that this does not exclude the existence of significantly faster angular momentum transfer processes, e.g. from orbital moment to the lattice, as long as this does not provide a bottleneck for angular momentum transfer as discussed below.

Comparing the spin and orbital moment dynamics at Fe and Gd sites, the different time constants of the element-specific angular momentum decay become apparent. Taking this into account, there is also no indication for a transfer of angular momentum between both sublattices during the first hundreds of femtoseconds. Otherwise, as Gd carries a much larger amount of magnetic moment per atom compared to Fe [20], one would expect to see a significant increase of demagnetization or even switching at Fe sites on the same time scale when Gd demagnetizes; this is obviously not the case - see Fig. 3. Note that it is also not 
possible to explain the observed loss of angular momentum by non-local processes like spin currents, which could transport angular momentum out of the probed area [23]. Because the magnetic layer is sandwiched between two insulating $\mathrm{Si}_{3} \mathrm{~N}_{4}$ layers and probed throughout its entire depth in transmission geometry, the observation of any demagnetization effect based on transport of magnetization out of the probing area, such as spin transport, can be ruled out. While a lateral spin transport can occur, it was shown by X-ray scattering experiments in Ref. [24] to happen only on the nanometer length scale, thus averaging out over our macroscopic probing volume, which was overfilled by the infrared pump beam. Thus, the observed loss of angular momentum can only be explained by a local process, involving a transfer to another reservoir.

The lack of an inter-atomic Fe-Gd exchange of angular momentum can be understood in terms of an electronic temperature dominated regime directly after laser excitation, when the electronic temperature of the system exceeds the Curie temperature, leading to a paramagnetic-like behavior with negligible exchange interaction between both sublattices [25]. By ruling out that the orbital moment at both Fe and Gd sites serves as an angular momentum reservoir, and because of the lack of angular momentum exchange between Fe and Gd on the observed time scales, the only remaining possibility is a full transfer of spin and orbital angular momentum to the lattice.

Recent theory work suggests the existence of such an ultrafast, spin-orbit mediated dissipation of angular momentum in ferromagnetic transition metals. By developing a manybody theory of ultrafast demagnetization, the authors in Ref. [11] demonstrate a transfer of angular momentum from spin to orbital moment on time scales of $\approx 10 \mathrm{fs}$, governed by the strength of the elemental spin-orbit interaction. In parallel, the transfer of the orbital momentum to the lattice is required by the fact that $L_{z}$ is not a conserved property at one atom in the solid, taking the interaction with neighboring atoms into account. In a Hubbard-like picture, the inter-atomic hopping integrals are on the order of $1 \mathrm{eV}$, resulting in a time constant of the order of 1 fs for the transfer of orbital angular momentum to the lattice. This mechanism can be viewed as a dynamic quenching of the orbital moment within the crystal field potential of the lattice. Given that this process is significantly faster than the spin-orbit mediated transfer into the orbital moment, no accumulation of orbital moment can thus be observed on the spin-orbit time scale [11], i.e. the spin-orbit interaction mediated step of angular momentum transfer from the spin moment to the orbital moment 
is the rate-limiting step in the flow of angular momentum to the lattice as the ultimate sink - i.e. the transfer from orbital moment to the lattice is not a bottleneck. Our experimental observations support this picture in that we see no accumulation of orbital angular momentum, nor a transfer of angular momentum between different elements on a sub-ps time scale. Our findings are in line with a very recent study showing that angular momentum generated upon laser-driven demagnetization of ferromagnetic Fe can indeed appear in the phonon system on a time scale of few hundred fs [26]. Future experiments with few-fs temporal resolution on conventional $3 \mathrm{~d} 4 \mathrm{f}$ magnets will be required to test if orbital angular momentum does indeed accumulate on this time scale. As the time scale is inversely proportional to electron hopping probabilities and hence also to the $3 \mathrm{~d}$ or $4 \mathrm{f}$ bandwidth, we suggest that the comparison of $L$ and $S$ demagnetization time constants of 3D and 2D magnetic systems may provide a route to directly witness the final disappearance of angular momentum from the electronic system.

We thank C. Schüßler-Langeheine, N. Pontius and V. Shokeen for the helpful discussions and support during our slicing experiments. Funding from German Ministry for Education and Research through the projects 05K16BCA, 05K16BCB and 05K10PC2 is gratefully acknowledged. A. Tsukamoto acknowledges support by the Grant-in-Aid for Scientific Research on Innovative Area, Nano Spin Conversion Science (Grant No. 26103004). We thank the Helmholtz-Zentrum Berlin for the allocation of synchrotron radiation beamtime.

*radu@mbi-berlin.de

[1] E. Beaurepaire, J.-C. Merle, A. Daunois, and J.-Y. Bigot, Phys. Rev. Lett. 76, 4250 (1996).

[2] A. Kirilyuk, A. V. Kimel, and T. Rasing, Rev. Mod. Phys. 82, 2731 (2010).

[3] C. D. Stanciu, F. Hansteen, A. V. Kimel, A. Kirilyuk, A. Tsukamoto, A. Itoh, and T. Rasing, Phys. Rev. Lett. 99, 047601 (2007).

[4] I. Radu, K. Vahaplar, C. Stamm, T. Kachel, N. Pontius, H. A. Durr, T. A. Ostler, J. Barker, R. F. L. Evans, R. W. Chantrell, A. Tsukamoto, A. Itoh, A. Kirilyuk, T. Rasing, and A. V. Kimel, Nature 472, 205 (2011).

[5] T. A. Ostler, J. Barker, R. F. L. Evans, R. W. Chantrell, U. Atxitia, O. Chubykalo-Fesenko, S. El Moussaoui, L. Le Guyader, E. Mengotti, L. J. Heyderman, F. Nolting, A. Tsukamoto, 
A. Itoh, D. Afanasiev, B. A. Ivanov, A. M. Kalashnikova, K. Vahaplar, J. Mentink, A. Kirilyuk, T. Rasing, and A. V. Kimel, Nature Communications 3, 666 (2012).

[6] K. Carva, P. Baláž, and I. Radu, Chapter 2 - Laser-Induced Ultrafast Magnetic Phenomena, edited by E. Brück, Handbook of Magnetic Materials, Vol. 26 (Elsevier, 2017) pp. 291 - 463.

[7] C. Stamm, T. Kachel, N. Pontius, R. Mitzner, T. Quast, K. Holldack, S. Khan, C. Lupulescu, E. Aziz, M. Wietstruk, H. Durr, and W. Eberhardt, Nature materials 6, 740 (2007).

[8] A. Einstein and W. J. de Haas, Deutsche Physikalische Gesellschaft 17 (1915).

[9] C. Boeglin, E. Beaurepaire, V. Halte, V. Lopez-Flores, C. Stamm, N. Pontius, H. A. Dürr, and J.-Y. Bigot, Nature 465, 458 (2010).

[10] N. Bergeard, V. Lopez-Flores, V. Halte, M. Hehn, C. Stamm, N. Pontius, E. Beaurepaire, and C. Boeglin, Nature Communications 5, 3466 (2014).

[11] W. Töws and G. M. Pastor, Phys. Rev. Lett. 115, 217204 (2015).

[12] K. Holldack, J. Bahrdt, A. Balzer, U. Bovensiepen, M. Brzhezinskaya, A. Erko, A. Eschenlohr, R. Follath, A. Firsov, W. Frentrup, L. Le Guyader, T. Kachel, P. Kuske, R. Mitzner, R. Müller, N. Pontius, T. Quast, I. Radu, J.-S. Schmidt, C. Schüßler-Langeheine, M. Sperling, C. Stamm, C. Trabant, and A. Föhlisch, Journal of Synchrotron Radiation 21, 1090 (2014).

[13] R. Abrudan, F. Brüssing, R. Salikhov, J. Meermann, I. Radu, H. Ryll, F. Radu, and H. Zabel, Review of Scientific Instruments 86, 063902 (2015).

[14] T. Kachel, F. Eggenstein, and R. Follath, Journal of Synchrotron Radiation 22, 1301 (2015).

[15] B. T. Thole, P. Carra, F. Sette, and G. van der Laan, Physical Review Letters 68, 1943 (1992).

[16] P. Carra, B. T. Thole, M. Altarelli, and X. Wang, Phys. Rev. Lett. 70, 694 (1993).

[17] Y. Teramura, A. Tanaka, and T. Jo, Journal of the Physical Society of Japan 65, 1053 (1996).

[18] Y. Teramura, A. Tanaka, B. T. Thole, and T. Jo, Journal of the Physical Society of Japan 65, 3056 (1996).

[19] K. Carva, D. Legut, and P. M. Oppeneer, EPL (Europhysics Letters) 86, 57002 (2009).

[20] I. Radu, C. Stamm, A. Eschenlohr, F. Radu, R. Abrudan, K. Vahaplar, T. Kachel, N. Pontius, R. Mitzner, K. Holldack, A. Föhlisch, T. A. Ostler, J. H. Mentink, R. F. L. Evans, R. W. Chantrell, A. Tsukamoto, A. Itoh, A. Kirilyuk, A. V. Kimel, and T. Rasing, SPIN 05, 1550004 (2015).

[21] U. Atxitia, J. Barker, R. W. Chantrell, and O. Chubykalo-Fesenko, Phys. Rev. B 89, 224421 
(2014).

[22] P. Bruno, Magnetismus von Festkörpern und Grenzflächen, edited by R. Hölzle (Forschungszentrum Jülich, 1993) Chap. 24.

[23] M. Battiato, K. Carva, and P. M. Oppeneer, Phys. Rev. Lett. 105, 027203 (2010).

[24] C. E. Graves, A. H. Reid, T. Wang, B. Wu, S. de Jong, K. Vahaplar, I. Radu, D. P. Bernstein, M. Messerschmidt, L. Müller, R. Coffee, M. Bionta, S. W. Epp, R. Hartmann, N. Kimmel, G. Hauser, A. Hartmann, P. Holl, H. Gorke, J. H. Mentink, A. Tsukamoto, A. Fognini, J. J. Turner, W. F. Schlotter, D. Rolles, H. Soltau, L. Strüder, Y. Acremann, A. V. Kimel, A. Kirilyuk, T. Rasing, J. Stöhr, A. O. Scherz, and H. A. Dürr, Nature Materials 12, 293 (2013).

[25] J. Mentink, J. Hellsvik, D. Afanasiev, B. A Ivanov, A. Kirilyuk, A. Kimel, O. Eriksson, M. Katsnelson, and T. Rasing, Physical review letters 108, 057202 (2012).

[26] C. Dornes, Y. Acremann, M. Savoini, M. Kubli, M. J. Neugebauer, E. Abreu, L. Huber, G. Lantz, C. A. F. Vaz, H. Lemke, E. M. Bothschafter, M. Porer, V. Esposito, L. Rettig, M. Buzzi, A. Alberca, Y. W. Windsor, P. Beaud, U. Staub, D. Zhu, S. Song, J. M. Glownia, and S. L. Johnson, Nature 565, 209 (2019). 